\title{
UNIFIED EIGENFILTER APPROACH: WITH APPLICATIONS TO SPECTRAL/SPATIAL FILTERING
}

\author{
Tsuhan Chen \\ Department of Electrical Engineering, 116-81 \\ California Institute of Technology \\ Pasadena, California 91125, USA
}

\begin{abstract}
In this paper, we extend the eigenfilter approach to solve general least-squares approximation problems with linear constraints. Such extension unifies previous work in eigenfilters and many other filter design problems, including spectral/spatial filtering, one-dimensional or multidimensional filters, data independent or statistically optimal filtering, etc. With this approach, various filter design problems are transformed into problems of finding an eigenvector of a positive definite matrix that is determined by filter design specifications. This approach has the advantage that many filter design constraints can be incorporated easily. A number of design examples are presented to show the usefulness and flexibility of the proposed approach.
\end{abstract}

\section{INTRODUCTION}

The eigenfilter approach for designing linear-phase FIR filters in the least-squares sense was introduced in [1]. This method minimizes a quadratic measure $E^{\prime}$ of the error in the passband and stopband, which can be expressed in the form $E=\mathbf{b}^{T} \mathbf{R} \mathbf{b}$. The matrix $\mathbf{R}$ is a real symmetric positive-definite matrix and the real vector $b$ is related to filter coefficients in a certain way. Under the constraint that $\mathbf{b}^{T} \mathbf{b}=1$, the filter coefficients contained in $\mathbf{b}$ are then computed by finding the eigenvector of $\mathbf{R}$ corresponding to its smallest eigenvalue. This approach requires very simple computation and has the advantage that both time and frequency domain constraints can be incorporated. The eigenfilter approach has been generalized to design filters having arbitrary response [2], Hilbert transformers and differentiators [3], [4], and two-dimensional (2D) filters [5], [6].

In this paper, we will apply the eigenfilter approach to general approximation problems. This will unify all

\footnotetext{
Work supported in parts by the NSF grant MIP 8919196 , and by matching funds from Tektronix, Inc., and Rockwell International.
}

previous work and extend the eigenfilter approach as well. Suppose we have a least-squares approximation problem together with some linear constraints. We will show that such a problem can be reformulated into a problem of minimizing $E=w^{\dagger} \mathbf{P w}$, where $w^{\dagger}$ denotes the transpose conjugate of the vector $w$, and $\mathbf{P}$ is a Hermitian positive-definite matrix. Then, the unknown vector w which minimizes $E$ can be found by computing the eigenvector of $\mathbf{P}$ corresponding to the smallest eigenvalue. We will also show how to incorporate various constraints into this minimization scheme. With such formulation, various kinds of filter design problems can be solved. These include: onedimensional (1D) and multidimensional (MD) filters, spectral/spatial filters with arbitrary sensor array geometry [7], [8], [9], statistically optimal filters [7], [8], etc. A number of design examples will be presented.

\section{EIGEN-APPROACH FOR LEAST-SQUARES APPROXIMATION PROBLEMS}

Consider the following approximation problem, which turns out to be general enough to cover many filter design problems. Suppose we want to approximate a given function $g(x)$ (possibly complex function) defined for real $x \in \mathcal{X}$, by using the linear combination of a set of $N$ functions $b_{0}(x), b_{1}(x), \ldots, b_{N-1}(x)$ which are also well-defined in $\mathcal{X}$. Let $w_{n}^{*}$, the conjugate of $w_{n}$, be the weights of such linear combination. With $w$ denoting the vector $\left[\begin{array}{lllll}w_{0} & w_{1} & \cdots & w_{N-1}\end{array}\right]^{T}$ and $\mathbf{b}(x)$ denoting $\left[b_{0}(x) b_{1}(x) \cdots b_{N-1}(x)\right]^{T}$, the result of such a linear combination can be expressed as

$$
f(x) \triangleq \sum_{n=0}^{N-1} w_{n}^{*} b_{n}(x)=\mathbf{w}^{\dagger} \mathbf{b}(x) .
$$

By properly choosing w, we want to make $f(x)$ 'close' to $g(x)$ for $x \in \mathcal{X}$. We now reformulate this problem into an eigenvector problem. First, we add the

0-7803-1254-6/93\$03.00 @ 1993 IEEE 
normalization constraint $\sum_{n=0}^{N-1}\left|w_{n}\right|^{2}=1$, or $\mathbf{w}^{\dagger} \mathbf{w}=$ 1. The purpose of this constraint will become clear later. Under this constraint, we have no control of the 'scale' of $f(x)$ any more. Therefore, we shall make $f(x) / f\left(x_{0}\right)$ approximate $g(x) / g\left(x_{0}\right)$, where $x_{0}$ is a reference point in $\mathcal{X}$. Equivalently, we minimize the following quadratic error measure

$$
E \triangleq \int_{x \in \mathcal{X}}\left|f(x)-f\left(x_{0}\right) \frac{g(x)}{g\left(x_{0}\right)}\right|^{2} d x .
$$

Using (1), we can rewrite $E$ as

$$
E=\int_{x \in \mathcal{X}}\left|\mathbf{w}^{\dagger} \mathrm{b}(x)-\mathbf{w}^{\dagger} \mathrm{b}\left(x_{0}\right) \frac{g(x)}{g\left(x_{0}\right)}\right|^{2} d x=\mathbf{w}^{\dagger} \mathbf{P w}
$$

where

$\mathbf{P}=\int_{x \in \mathcal{X}}\left[\mathbf{b}(x)-\mathbf{b}\left(x_{0}\right) \frac{g(x)}{g\left(x_{0}\right)}\right]\left[\mathbf{b}(x)-\mathbf{b}\left(x_{0}\right) \frac{g(x)}{g\left(x_{0}\right)}\right]^{\dagger} d x$.

We can see that $\mathbf{P}^{\dagger}=\mathbf{P}$, i.e., $\mathbf{P}$ is a Hermitian matrix. Also, the error $E=\mathbf{w}^{\dagger} \mathbf{P w}$ is positive for any nonzero $\mathbf{w}$, so $\mathbf{P}$ is positive-definite. (It can be positivesemidefinite if there exists w such that $E=0$, which implies that the approximation can be made exact.) Therefore, all the eigenvalues of $\mathbf{P}$ are real and positive. According to the Rayleigh-Ritz theorem [10], the vector w which minimizes $E=\mathbf{w}^{\dagger} \mathbf{P} \mathbf{w}$ under the constraint $\mathbf{w}^{\dagger} \mathbf{w}=1$ is the eigenvector of $\mathbf{P}$ corresponding to the smallest eigenvalue. Such eigenvector can be computed using the power method [10], [1]. When the ratio between the two smallest eigenvalues of $\mathbf{P}$ is sufficiently large, this method converges very fast. Hence, the required computation complexity is relatively low, compared with other approximation schemes which usually involve matrix inversion operations.

Scaling $f(x)$ : Since we have made $f(x) / f\left(x_{0}\right)$ approximate $g(x) / g\left(x_{0}\right)$, we should scale the resulting $f(x)$ properly to get the final solution. We can see that the final solution which approximates $g(x)$ should be $f(x) g\left(x_{0}\right) / f\left(x_{0}\right)$.

The choice of $x_{0}:$ In (2), we need to choose $x_{0}$ such that $g\left(x_{0}\right) \neq 0$. If $g(x)=0$ for all $x$ in $\mathcal{X}$, we simply let $E=\int_{x \in \mathcal{X}}|f(x)|^{2} d x$, which can be written as

$$
E=\int_{x \in \mathcal{X}}\left|\mathbf{w}^{\dagger} \mathbf{b}(x)\right|^{2} d x=\mathbf{w}^{\dagger} \mathbf{P} \mathbf{w}
$$

where $\mathbf{P}=\int_{x \in \mathcal{X}} \mathbf{b}(x) \mathbf{b}(x)^{\dagger} d x$. Then, this becomes a quadratic form which can be minimized by finding the eigenvector with the smallest eigenvalue.

Generalization of (2): The variable $x$ may be a $1 D$ scalar or an MD vector. For the MD case, the integral in (2) should be rewritten as a multiple integral. The variable $x$ can also be a discrete variable, and hence $\mathcal{X}$ becomes a discrete domain. In this case, the integral of (2) should be replaced by a summation.

\section{INCORPORATING OTHER CONSTRAINTS}

The main advantage of the eigenfilters is that various time or frequency domain constraints can be incorporated [1]. We will show that the generalized eigenapproach mentioned in the previous section has the same advantage.

For example, suppose we want to minimize the approximation error $E$ and at the same time minimize some other quadratic form of $\mathbf{w}$, say $\mathbf{w}^{\dagger} \mathbf{Q} \mathbf{w}$ where $\mathbf{Q}$ is Hermitian and positive-definite. We can achieve this by minimizing

$$
\begin{aligned}
E_{1} & =(1-\alpha) \mathbf{w}^{\dagger} \mathbf{P} \mathbf{w}+\alpha \mathbf{w}^{\dagger} \mathbf{Q w} \\
& =\mathbf{w}^{\dagger} \underbrace{[(1-\alpha) \mathbf{P}+\alpha \mathbf{Q}]}_{\mathbf{S}} \mathbf{w}
\end{aligned}
$$

where the parameter $\alpha$ controls the weight of this additional constraint. Then, we simply compute the eigenvector of $\mathbf{S}$ with the smallest eigenvalue.

In some occasions, we want to minimize the approximation error $E$ with some linear constraints on $\mathbf{w}$. With $M$ denoting the number of constraints, we can express these constraints as $\mathbf{C w}=\mathbf{d}$, where $\mathbf{C}$ is a $M \times N$ matrix and $\mathbf{d}$ is an $M \times 1$ vector.

\section{Case 1: $\mathbf{d}=\mathbf{0}$}

All the vector $\mathbf{w}$ satisfying $\mathbf{C w}=\mathbf{0}$ can be written as $\mathbf{w}=\mathbf{F a}$, where the columns of $\mathbf{F}$ form a basis of the subspace orthogonal to the row space of $\mathbf{C}[11]$. So, minimizing $\mathbf{w}^{\dagger} \mathbf{P w}$ under the constraint $\mathbf{C w}=\mathbf{0}$ is the same as minimizing $\mathbf{a}^{\dagger} \mathbf{F}^{\dagger} \mathbf{P F a}$ without any constraints. Hence, we only have to compute the eigenvector of $\mathbf{F}^{\dagger} \mathbf{P F}$. Instead of doing this, we can also minimize the energy of $\mathbf{C w}$, which is $|\mathbf{C w}|^{2}=\mathbf{w}^{\dagger} \mathbf{C}^{\dagger} \mathbf{C w}$. This becomes a quadratic form that can be incorporated as in (6).

\section{Case 2: $\mathbf{d} \neq 0$}

For this case, instead of satisfying $\mathbf{C w}=\mathbf{d}$ exactly, we can try to approximate $\mathbf{d}^{*}$ using $\mathbf{C}^{*} \mathbf{w}^{*}$, the linear combination of columns of $\mathbf{C}^{*}$. Then, this constraint itself becomes an approximation problem. To see this, let $\mathcal{X}$ be the discrete domain $\{0,1, \ldots, M-1\}, b_{n}(x)$ be the $(x, n)$-th element of $\mathbf{C}^{*}$, and $g(x)$ be the $x$-th 
element of $\mathbf{d}^{*}$. Using the scheme described in Section 2 , we can approximate $g(x)$ with

$$
f(x)=\sum_{n=0}^{N-1} w_{n}^{*} b_{n}(x)=\sum_{n=0}^{N-1} w_{n}^{*}\left[\mathbf{C}^{*}\right]_{x n}=\mathbf{C}^{*} \mathbf{w}^{*}
$$

\section{APPLICATIONS TO FHLTER DESIGN AND DESIGN EXAMPLES}

By properly choosing the functions $b_{n}(x)$, various filter design problems can be formulated into least-squares approximation problems. For example, letting $b_{n}(x)$ be $\cos (\omega n)$, we can design Type 1 linear phase FIR filters [1]. For the $2 \mathrm{D}$ case, taking $x$ to be the $2 \mathrm{D}$ vector $\left[\begin{array}{ll}\omega_{0} & \omega_{1}\end{array}\right]^{T}$, and letting $b_{n}(x)$ be $\cos \left(\omega_{0} n_{0}\right) \cos \left(\omega_{1} n_{1}\right)$, we obtain the 2D linear-phase eigenfilter approach as described in [5] and [6]. Therefore, previous $1 D$ or 2D eigenfilter approaches are indeed unified by the generalized eigen-approach.

Spatial filtering, which finds applications in beamforming [7], [8], [9] can also be achieved using the eigenapproach. All we need to do is to find the appropriate functions $b_{n}(x)$. We now present two examples to show this.

\section{Design Example 1: Narrow-band linear array}

Consider a linear array having $N$ sensors spaced at one-half wavelength as shown in Fig. 1 . The output of the $n$-th sensor is weighted by $w_{n}^{*}$ to produce the overall output. The gain of this sensor array with respect to a uniform plane wave arriving at angle $\theta$ is

$$
f(\theta)=\sum_{n=0}^{N-1} w_{n}^{*}[\exp (-j n \pi \sin \theta)]=\mathbf{w}^{\dagger} \mathbf{b}(\theta) .
$$

So, the appropriate $b_{n}(x)$ functions for this problem are $b_{n}(\theta)=\exp (-j n \pi \sin \theta)$. In fact, the vector $\mathbf{b}(\theta)$ obtained above is the so-called steering vector or direction vector [7], [8]. Then, we can use the eigen-approach to compute the proper weights $w_{n}^{*}$. For the case $N=16$, suppose we want to 'steer' the array to an arrival angle of $20^{\circ}$. We can achieve this by constraining the gain to be unity for $\theta=20^{\circ}$ and minimizing the energy elsewhere using the eigen-approach. Fig. 2 shows the resulting gain versus $\theta$. Similar to [1], it is possible to make the response equiripple by putting proper weighting function in (2).

Design Example 2: Arc array with delay lines

An array with attached delay lines can provide both spatial and spectral filtering. In general, the response of such an array, which is a function of both the arrival angel $\theta$ and the frequency $\omega$, can be expressed as $f(\theta, \omega)=\mathbf{w}^{\dagger} \mathbf{b}(\theta, \omega)$. The vector $\mathbf{b}(\theta, \omega)$ is

$$
\mathbf{b}(\theta, \omega)=\left[\begin{array}{lll}
e^{j \omega \tau_{0}(\theta)} & e^{j \omega \tau_{1}(\theta)} \ldots e^{j \omega \tau_{N-1}(\theta)}
\end{array}\right],
$$

where $\tau_{n}(\theta)$ is the time delay (due to propagation and tap delays) of point at which the weight $w_{n}^{*}$ is applied. Consider the case of an arc array with delay lines, as shown in Fig. 3, where $R$ is the radius of the arc and $\alpha_{m}$ denotes the angle of the $m$-th sensor. It can be shown that

$$
\tau_{m, \ell}(\theta)=-\frac{R}{c}\left[\cos \theta-\cos \left(\alpha_{m}-\theta\right)\right]-\ell T_{s}
$$

for the $\ell$-th tap of the $m$-th sensor. In this expression, $c$ is the speed of the wave, and $T_{s}$ is the unit delay in the delay lines. In our experiment, we choose an arc array with ten sensors, each attached with ten taps. The $\alpha_{m}$ are chosen to be $0^{\circ}, 2^{\circ}, 4^{\circ}, 6^{\circ}, 8^{\circ}, 10^{\circ},-2^{\circ},-5^{\circ},-8^{\circ}$, and $-11^{\circ}$. Note that the sensors do not have to be equi-spaced for the eigen-approach to work. Using the eigen-approach, we can approximate the response to be unity in the range $41^{\circ} \leq \theta \leq 49^{\circ}$ and $0.4 \pi \leq \omega \leq 0.8 \pi$ and minimize the response around $\theta=0^{\circ}$ (e.g., if we want to suppress some interference coming from that angle). Fig. 4 shows the response resulting from the optimized weights. Although we show only the case of data independent filtering, statistically optimal filtering can be achieved similarly.

\section{CONCLUSIONS}

In this paper, we have proposed a generalized eigenfilter approach which unifies all previous work in eigenfilters. Due to its generality, many filter design problems, 1D or $\mathrm{MD}$, spectral or/and spatial, data independent or statistically optimal, can be solved using this method. We have also presented a number of design examples to illustrate the usefulness and flexibility offered by the proposed approach.

\section{REFERENCES}

[1] P. P. Vaidyanathan and T. Q. Nguyen, "Eigenfilters: a new approach to least-squares FIR filter design and applications including Nyquist filters," IEEE Trans. on CAS, pp. 11-23, January 1987.

[2] T. Q. Nguyen, "The eigenfilter for the design of linear-phase filters with arbitrary magnitude response," IEEE ICASSP, Canada, May 1991.

[3] S.-C. Pei and J.-J. Shyu, "Design of Hilbert transformers and differentiators by eigenfilters," IEEE Trans. on Circuits and Systems, vol. 35, no. 11, pp. 1457-1461, November 1988. 
[4] S.-C. Pei and J.-J. Shyu, "Eigenfilter design of higher-order digital differentiators," IEEE Trans. on Acoustics, Speech, and Signal Processing, vol. 37, pp. 505-511, April 1989.

[5] A. Nashashibi, and C. Charalambous, "2-D FIR eigenfilters," Proc. IEEE Int. Symp. Circuits and Systems, Finland, pp. 1037-1040, June 1988.

[6] S.-C. Pei and J.-J. Shyu, "2-D FIR eigenfilters: a least-squares approach," IEEE Trans. on CAS, pp. 24-34, January 1990.

[7] K. M. Buckley, "Spatial/spectral filtering with linearly constrained minimum variance beamformers," IEEE Trans. on Acoustics, Speech, and Signal Processing, vol. 35, pp. 249-266, March 1987.

[8] B. D. Van Veen and K. M. Buckley, "Beamforming: a versatile approach to spatial filtering ," IEEE ASSP Magazine, pp. 4-24, April 1988.

[9] W. H. Kummer, "Basic array theory," Proc. IEEE, vol. 80, no. 1, pp. 127-140, January 1992.

[10] R. A. Horn and C. R. Johnson, Matrix Analysis, Cambridge University Press, 1985.

[11] S. H. Friedberg, A. J. Insel, and L. E. Spence, Linear Algebra, Prentice-Hall, 1979.

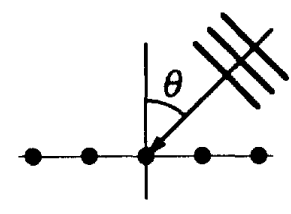

Fig. 1. A linear array.

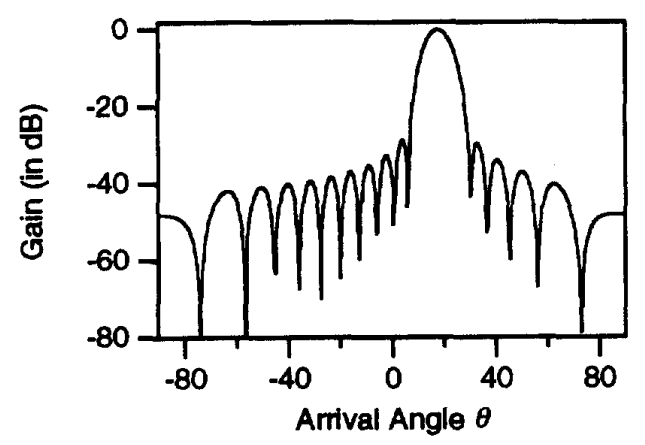

Fig. 2. Gain of a linear array.

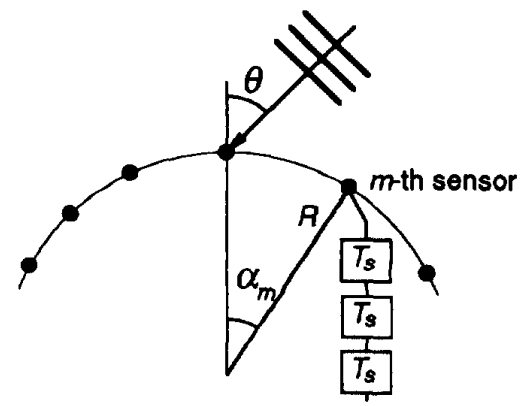

Fig. 3. An arc array with delay lines.
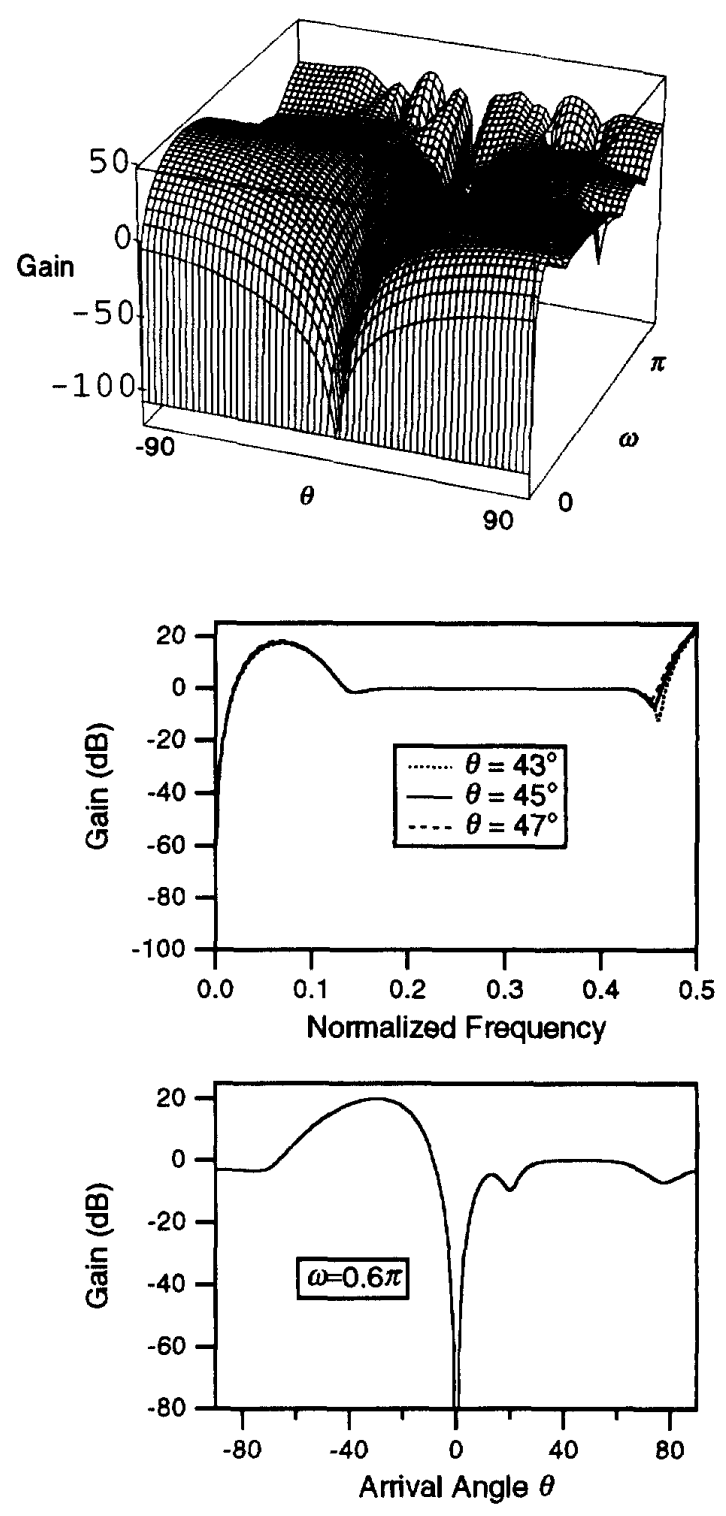

Fig. 4. Gain of an arc array with delay lines. 